\title{
Wavefront Modulation for an Elongated Depth of Focus with a Homogeneous Point Spread Function
}

\author{
Daniel Iwaniuk and Erwin Hack \\ Laboratory of Electronics/Metrology/Reliability, Empa, Überlandstr. 129, 8600 Dübendorf, Switzerland.
}

\begin{abstract}
We present a pure phase pupil filter design for an extended depth of focus (DoF) with a homogeneous transversal behavior. State of the ort solutions base on complex (amplitude and phase) modulation or on diffractive phase filters, which have either low conversion efficiency due to the blocking of light or very complex structures. Our design is a quartic multiplex phase-only function with good performance and simple annular structure. Numerical simulation based on the Kirchhoff diffraction integral and experiments performed with a modulation transfer function measurement system combined with a spatial phase modulation liquid crystal device show in agreement a three times enlarged focal depth with homogeneous transversal spot size. Further simulations suggest that the depth of focus can be extended up to a factor 12 . However, when using monochromatic polarized light interference effects disturb the uniform intensity along the focal depth..
\end{abstract}

Keywords: Liquid crystal device (LCD), phase pupil filter, elongated depth of focus, PSF, transversal gain. PACS: 42.79.CI, 42.15.Eq

\section{INTRODUCTION}

High depth of focus (DoF) systems have a wide application area covering optical data storage, endoscopic optical coherence tomography, microscopy, video imaging, ophthalmology or optical microlithography. There exist many different designs to generate an elongated DoF without changing the numerical aperture (NA) [1-5]. The principle of pupil filters is to modulate the amplitude or phase of the incoming light in the lens plane (pupil plane) to engineer the focusing properties of the lens. So far a homogeneous transversal distribution over the complete DoF was achieved only by amplitude modulation or by complex diffractive filters. They possess several disadvantages like low light efficiency due to the blocking of light or difficult production of the complicated diffractive structures. Therefore, we focus our investigations on pure phase filters with at least piecewise continuous modulation. Continuous quartic polynomial phase functions produce an elongation of the axial spot size, but show an inhomogeneous transversal behavior. We present simulations and experimental implementation of an annular quartic multiplex function, which is easy to produce and shows a constant transversal distribution over the DoF.

The numerical simulations base on the well known scalar diffraction theory that is valid for low NA systems as used in this work. The main quantity of interest is the transversal behavior within the depth of focus, but we also use other figures of merit such as the Strehl ratio, FWHM in axial and transversal direction for comparison with the experiments.. The experiments are performed with a typical Modulation Transfer Function (MTF) measurement system in combination with a phase-only spatial light modulator (SLM).

Results from numerical simulations and optical experiments show, that with conventional pure phase filters the transversal point spread function (PSF) spot size (resolution) is quite inhomogeneous over the DoF. Our quartic multiplex phase function is able to generate a homogeneous transversal resolution over the DoF with a size similar to the reference resolution, but shows interference effects in the axial direction. 


\section{METHOD: SIMULATION \& EXPERIMENT}

\section{Numerical Calculations \& Pupil Filter Design}

The normalized light intensity near the focus $(r=0, z=0)$ of a lens with focal length $f$ is [6]:

$$
I(r, z)=\left\{\frac{k}{z} \int_{0}^{\rho_{\max }} P(\rho) \cdot e^{i \frac{k \rho^{2}}{2 f}} \cdot e^{i \frac{k \rho^{2}}{2 z}} \cdot J_{0}\left(\frac{k}{z} \rho r\right) \cdot \rho \cdot d \rho\right\}^{2}
$$

where $\mathrm{k}=2 \pi / \lambda$ is the wave number, $\rho$ the radial aperture in the pupil plane, $\mathrm{P}(\rho)$ the radial pupil filter and $\mathrm{J}_{0}$ the Bessel function of order zero. In this work, a laser wavelength of $\lambda=532 \mathrm{~nm}$, an aperture radius of $\rho=5 \mathrm{~mm}$ and a focal length of $\mathrm{f}=100 \mathrm{~mm}$ was used.

The figures of merit are extracted from the intensity distribution. The Strehl ratio $\mathrm{S}$ is the maximal intensity value with a defined value of 1 for a diffraction limited lens with the same focal length. The depth of focus (DoF) is the FWHM of the axial intensity distribution. Axial and transversal gain factors $G_{A}$ and $G_{T}$ are defined as the ratio of the corresponding FWHM and the reference value from the diffraction limited lens. A gain factor larger than 1 results in a broadening of the spot, while a gain factor smaller than 1 represents a narrowing.

The pupil filter design bases on the formulation in equation 2, describing a quartic function [7]:

$$
P_{ \pm}(\rho)=\exp \left(-1 / 2 \mathrm{ik}\left(A_{1} \cdot \rho^{2} \pm A_{2} \cdot \rho^{4}\right)\right)
$$

The parameter $A_{1}$ shifts the focal length, while $A_{2}$ elongates the DoF. However, it shows a broadening effect on one end and a narrowing effect on the other as shown in the results section. Our design combines two opposite quartic functions in an annular multiplexed way. Therefore, two quartic functions with positive and negative $\mathrm{A}_{2}$ but similar axial gain are axially shifted using the corresponding parameters $A_{1}$ until they overlap. Some 10-20 radial zones are defined in the aperture plane with alternating quartic function $\mathrm{P}_{ \pm}$, resulting in a zone width of $0.5-0.25$ $\mathrm{mm}$.

\section{Experimental Setup}
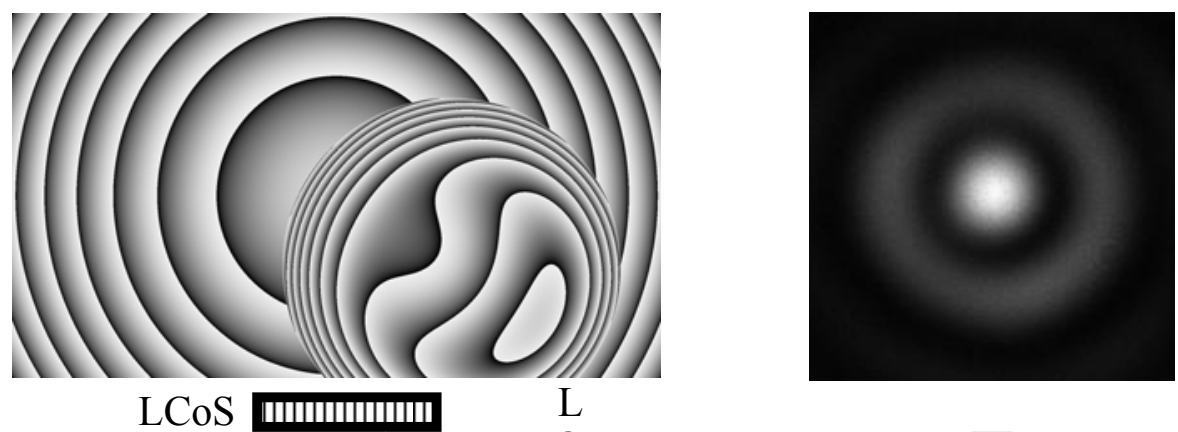

$\mathrm{L}$

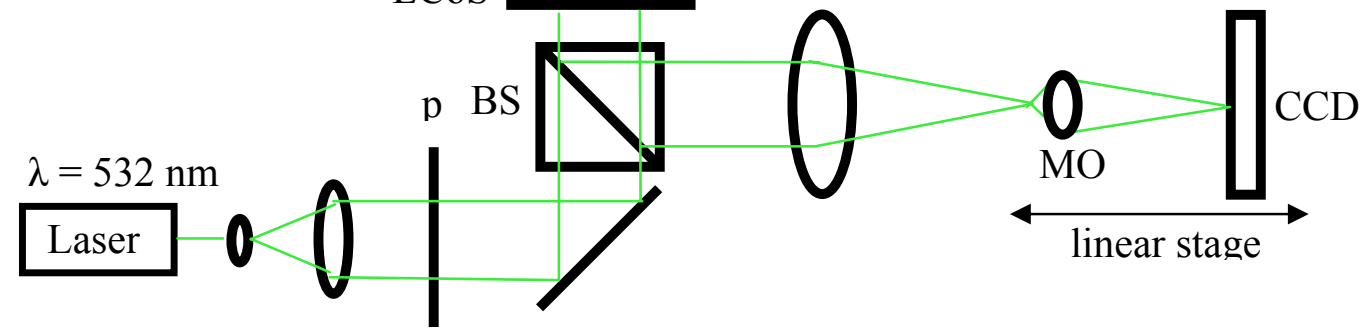

FIGURE 1, Experimental scheme (p: polarizer, BS: beam splitter, LCoS: liquid crystal on silicon, L: lens, MO: microscope objective, CCD: camera). Left picture: pupil function and flatness correction overlaid, right picture: PSF measured on CCD. 
The experimental scheme is presented in figure 1. A green laser (Nd:YAG Crystalaser, wave length $532 \mathrm{~nm})$ is expanded and its polarization set to the axis of a reflective liquid crystal spatial light modulator (Pluto from Holoeye with 1980x1080 pixel resolution). The phase function of the pupil filter is applied in addition to a correction of the curvature of the device (top left in figure 1). The modulated beam is passed to a lens. Its focal region is magnified with a microscope objective with higher NA onto a CCD camera (top right in figure 1). The image analyzer is positioned on a translation state, allowing measurements of the complete 3D PSF.

\section{RESULTS:}

The results are split in two parts. The first sub part shows simulation and experiments to achieve an axial gain of 3. Then numerical simulations show that the quartic multiplex filter is also able to generate much higher axial gains.

\section{Axial Gain 3: Simulation \& Experiment}

Four different designs are presented in the figures: the unmodulated lens with $100 \mathrm{~mm}$ focal length (black solid), two opposite quartic functions $\mathrm{P}_{+}$(green cyrcles) and $\mathrm{P}_{-}$(blue squares), and the quartic multiplex function (red crosses). The increase in DoF is set to around 3 for each design.

The simulations and experiment show in agreement, that a simple quartic function design produces an inhomogeneous transversal behavior within the elongated focus. There is an apodization at one end of the DoF and a superresolution effect at the other end, while the central region has a resolution similar to the reference (unmodified) lens (figures $2 \& 3$ ). Simulations show a transversal gain difference between 0.7 and 2.1 for both functions, but apparently mirrored. The experimental values lie within 0.8 and 3.1 for the $\mathrm{P}_{+}$and within 0.9 and 2.9 for the $\mathrm{P}_{\text {. }}$ design. By combining two opposite phase functions with similar DoF to a quartic multiplex, the transversal distribution gets homogeneous while the axial distribution remains elongated. In the simulation, the transversal gain has a constant value of 1 over the complete DoF and the small side lobes outside the DoF. The experimental values fluctuate around a transversal gain of $1.5 \pm 0.5$, but remain low at both end of the DoF and within the side lobes. Simulation and experiment prove that it is possible to generate a constant transversal gain within an elongated focus with a pure phase filter. There exist diffractive phase filters [8] or complex pupil filters [9] with good performance as well. They have much more complex structures or very low Strehl values, respectively.

The observed differences between the experiment and the simulation are attributed to the imperfect correction of the bent surface of the SLM, imperfections of the lens, and misalignment between the laser beam and the modulation function on the LCD. The increased side lobes of the quartic functions (blue squares and green cyrcles in figure 3) show typical sixth order aberrations. The reason of the inhomogeneity of the transversal gain of the quartic multiplex (red crosses) is unknown yet, but might be a combination of the above mentioned reasons. However, the predicted performance of the simulation was successfully shown in the experiment. 


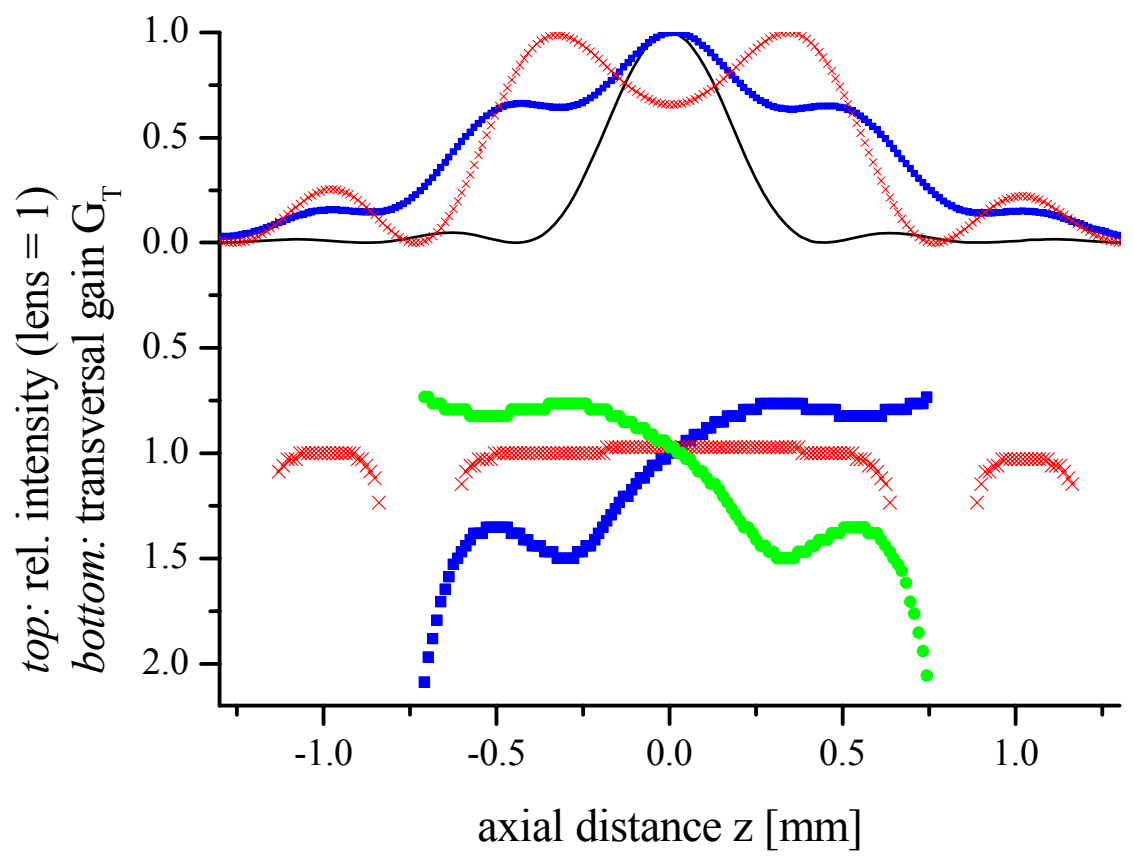

FIGURE 2, Numerical simulation for different pupil filter designs (black solid: reference without modulation, blue squares: P-, green cyrcles: $\mathrm{P}+$, red crosses: quartic multiplex). Top: axial intensity distribution, bottom: transversal gain at each position with a higher Strehl value than one quarter. (Note: the blue squares and green cyrcles intensity distributions are overlapping)

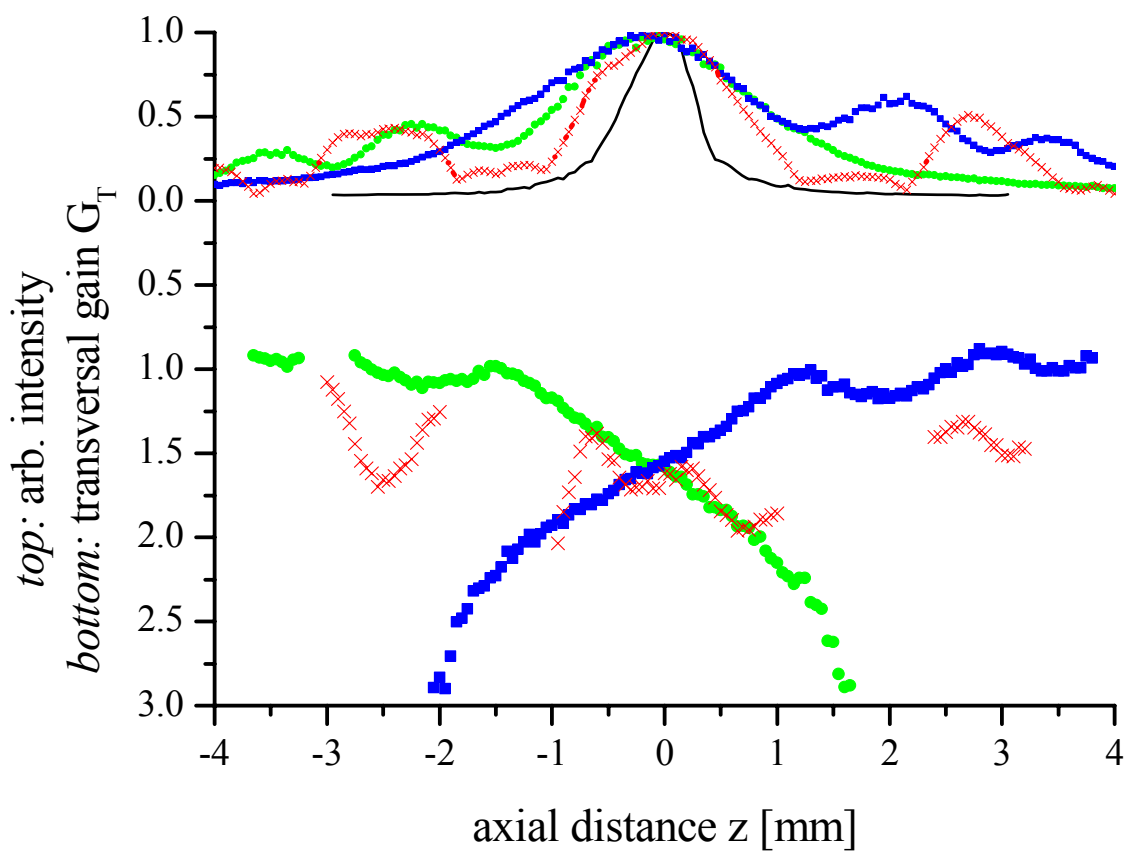

FIGURE 3, Experimental results for different pupil filter designs (black solid: reference without modulation, blue squares: P-, green cyrcles: $\mathrm{P}+$, red crosses: quartic multiplex). Top: axial intensity distribution, bottom: transversal gain at each position with a Strehl value higher than 0.25 . 


\section{Axial Gain 12: Simulation}

Further investigations with the same basic parameters but with higher DoF were performed. The fourth order parameter $\mathrm{A}_{2}$ was changed in the numerical simulations to obtain an axial gain factor of 12 (figure 4). The quartic functions $\mathrm{P}_{ \pm}$(green cyrcles and blue squares) behave exactly the same as previously with higher narrowing and apodization at the ends. The axial intensity distribution of the quartic multiplex function (red crosses) shows strong interferences. The transversal gain is again constant over the complete region. The numerical simulations base on monochromatic polarized light. We expect that the interference effects diminish under white light illumination.

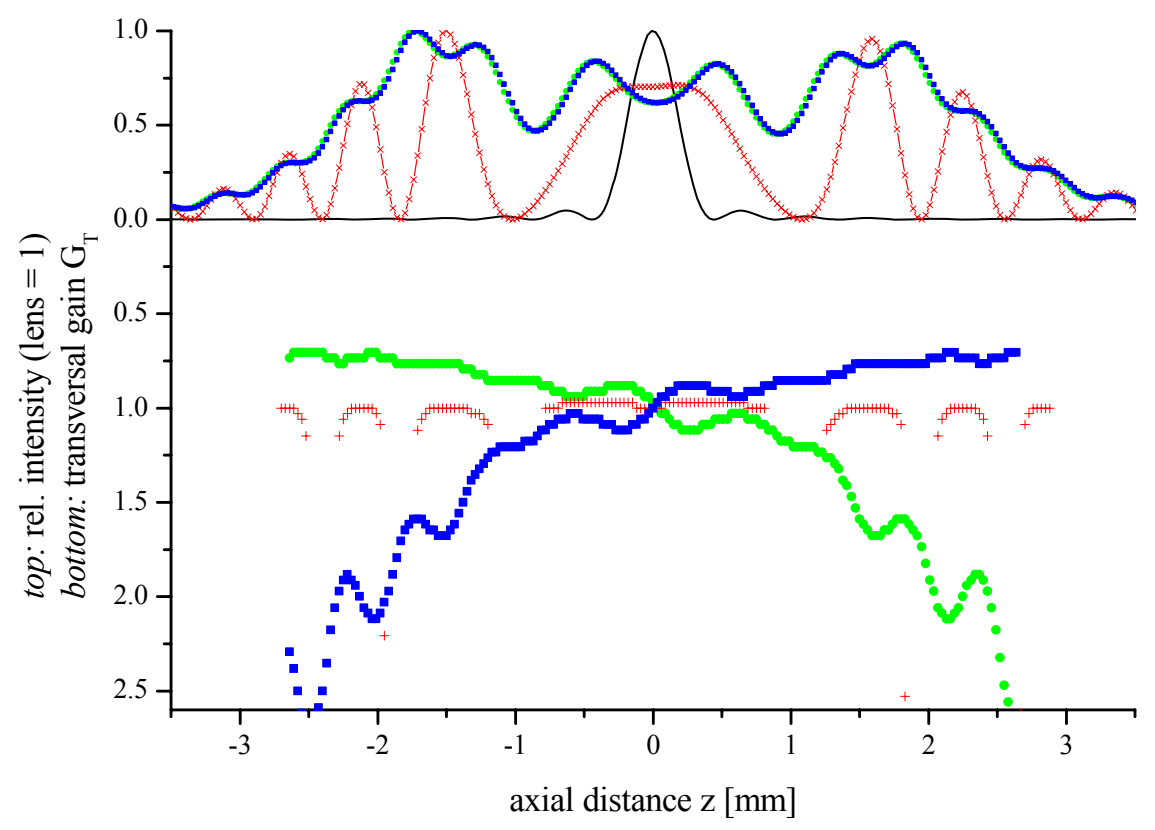

FIGURE 4, Numerical simulation with an axial gain of 12 (same properties as in figure 2).

\section{ACKNOWLEDGEMENT}

This research is partly funded by the Swiss National Science Foundation project Nr. 200021-116414.

\section{REFERENCES}

1. A. Burvall, K. Kolacz, Z. Jaroszewicz, and A. T. Friberg, "Simple lens axicon," Appl. Optics 43, 4838-4844 (2004).

2. Z. Q. Liu, A. Flores, M. R. Wang, and J. J. Yang, "Diffractive infrared lens with extended depth of focus," Opt. Eng. 46, 9 (2007).

3. C. J. R. Sheppard, J. Campos, J. C. Escalera, and S. Ledesma, "Three-zone pupil filters," Optics Communications 281, 3623-3630 (2008).

4. C. Iemmi, J. Campos, J. C. Escalera, O. Lo?pez-Coronado, R. Gimeno, and M. J. Yzuel, "Depth of focus increase by multiplexing programmable diffractive lenses," Opt. Express 14, 10207-10219 (2006).

5. J. A. Garcia, S. Bara, M. G. Garcia, Z. Jaroszewicz, A. Kolodziejczyk, and K. Petelczyc, "Imaging with extended focal depth by means of the refractive light sword optical element," Opt. Express 16, 18371-18378 (2008).

6. J. W. Goodman, Introduction to Fourier optics (McGraw-Hill, New York etc., 1996).

7. J. Rosen, B. Salik, and A. Yariv, "Pseudo-nondiffracting beams generated by radial harmonic functions," Journal of the Optical Society of America A: Optics and Image Science, and Vision 12, 2446-2457 (1995).

8. A. Flores, M. R. Wang, and J. J. Yang, "Achromatic hybrid refractive-diffractive lens with extended depth of focus," Appl. Optics 43, 5618-5630 (2004).

9. J. A. Davis, C. S. Tuvey, O. Lopez-Coronado, J. Campos, M. J. Yzuel, and C. Iemmi, "Tailoring the depth of focus for optical imaging systems using a Fourier transform approach," Optics Letters 32, 844-846 (2007). 